\title{
Solving Close-Open Mixed Vehicle Routing Problem Using Bat Algorithm
}

\author{
Atika Dwi Hanun Amalia ${ }^{1}$, Herry Suprajitno ${ }^{2}$ \& Asri Bekti Pratiwi ${ }^{3}$ \\ 1,2,3 Mathematics Department, Faculty of Science and Technology, Universitas Airlangga \\ ${ }^{2}$ Corresponding author: herry-s@fst.unair.ac.id
}

\begin{abstract}
The purpose of this research is to solve the Close-Open Mixed Vehicle Routing Problem (COMVRP) using Bat Algorithm. COMVRP which is a combination of Close Vehicle Routing Problem or commonly known as Vehicle Routing Problem (VRP) with Open Vehicle Routing Problem (OVRP) is a problem to determine vehicles route in order to minimize total distance to serve customers without exceed vehicle capacity. COMVRP occurs when the company already has private vehicles but its capacity could not fulfill all customer demands so the company must rent several vehicles from other companies to complete the distribution process. In this case, the private vehicle returns to the depot after serving the last customer while the rental vehicle does not need to return. Bat algorithm is an algorithm inspired by the process of finding prey from small bats using echolocation. The implementation program to solve was created using Java programming with NetBeans IDE 8.2 software which was implemented using 3 cases, small data with 18 customers, medium data with 75 customers and large data with 100 customers. Based on the implementation results, it can be concluded that the more iterations, the smaller total costs are obtained, while for the pulse rate and the amount of bat are tends not to affect the total cost obtained.
\end{abstract}

Keywords: bat algorithm, close-open mixed vehicle routing problem.

\section{Introduction}

Nowadays, rapid progress in production, transportation and technology has made the industrial sector more competitive. So to compete, a company must pay attention to many factors in order to survive and be able to get the maximum profit. One factor that can be maximized is distribution. Distribution is the process to supply goods or services from producers to consumers spread quickly, accurately and effectively. Distribution activities are useful for bringing producers closer to consumers or as intermediaries between production and consumption activities [1].

In distribution, determine the right route has big influence in the success of the distribution process because distribution with minimal distance and being able to serve all customer requests can make more profits for the company [2]. One type of distribution route determination problem is called Close Vehicle Routing Problem (Close-VRP) or commonly called Vehicle Routing Problem (VRP). The VRP problem can be defined as the problem of finding a route with a minimum cost from a depot to an agent whose location is spread out with different number of requests. In VRP, all routes start and end 
at the depot, each customer must be served exactly once with only one vehicle and the number of requests in one route may not exceed the capacity of the vehicle [3]. One type of VRP is the Close-Open Mixed Vehicle Routing Problem (COMVRP) which is a combination of Close-VRP and Open-VRP. COMVRP can be used in determining the distribution route of a company that has several private vehicles, but the vehicle is not able to meet all customer demands, resulting in the company having to rent several vehicles from other companies to meet customer demand. In this case, the private vehicle will return to the depot after serving the last customer while the rental vehicle will not return to the depot [4].

Bat Algorithm (BA) is one of the algorithms introduced by Xin-She Yang in 2010. In general, the bat algorithm mimics the behavior of bats in searching for food using emitted sound waves. In the bat algorithm there are three approaches used, the first of all bats using echolocation to estimate distance and distinguish between prey or obstacles. Then, each bat flies with random speed and position and can automatically adjust the frequency of the sound waves it emits and adjust the pulse rate depending on the proximity to the target. And finally, the level of loudness is assumed to start from the positive value of the largest constant to the smallest constant value [5]. According to Yang (2010), Bat Algorithm (BA) shows better performance than Particle Swarm Optimization (PSO), Genetic Algorithm (GA) or Harmony Search (HS).

In this article, the settlement of the Close-Open Mixed Vehicle Routing Problem (COMVRP) using the Bat Algorithm which aims to form an optimal route for a number of vehicles to a number of customers will be organized as follows, section 2 discussing close-open mixed vehicle routing problem. Section 3, Bat algorithm will be explained. Then section 4 is about the solution of COMVRP using bat algorithm. Afterwards, section 5 is computational result of COMVRP using bat algorithm in example case. Section 6 is conclusion. Finally, section 7 is suggestion for future research.

\section{Close-Open Mixed Vehicle Routing Problem}

Close-Open Mixed Vehicle Routing Problem (COMVRP) is a variation of Vehicle Routing Problem (VRP) which is a combination of the theory of Close-VRP and OpenVRP. Problems Close-Open Mixed Vehicle Routing Problem (COMVRP) occurs when a company that will distribute the goods to the customer but the customer demand exceeds the total capacity of private vehicles, thus requiring the company to hire some vehicles that customer demand can be met [6]. In COMVRP every trip made by a private vehicle starts and ends at the depot while every trip made by a leased vehicle starts from the depot and ends at the last customer.

Mathematically, COMVRP is expressed as a complete graph $\mathrm{G}$, for example graph $G=$ $(V, E)$, is a complete graph with $V=\{0\} \cup N$ a is a set of points indicating the distribution location, where $N=\{1,2, \ldots, n\}$ indicates the location of the customer, while point 0 
indicates the location of the depot. $E=\{(i, j): i, j \in V, i \neq j\}$ are lines that indicate the connecting road of the customer's location. The cost of travel between customer locations is $c_{i j}$, with provisions $c_{i j}=c_{j i}$.

In COMVRP, there are two types of vehicles used, they are private vehicles and rental vehicles. The set of private vehicles is $\mathrm{K} 1$ where $\mathrm{K} 1=\{1,2, \ldots, N u\}$ with $\mathrm{Nu}$ is the maximum number of private vehicles and the set of rental vehicles is $\mathrm{K} 2$ where $\mathrm{K} 2=$ $\{N u+1, N u+2, N u+3, \ldots\}$ the number of rental vehicles is assumed to be unlimited. For each vehicle $k$ there is a vehicle capacity of Maxcapacity and for each customer $j$ there is a demand of $q_{j}$.

The mathematical model according to Liu and Jiang [4] is formulated as follows:

$$
\operatorname{Min} Z=\sum_{k \in K} F_{k} \sum_{i \in N} x_{0 i}^{k}+\sum_{k \in K} \sum_{i \in N} \sum_{j \in N} c_{i j} x_{i j}^{k}-\sum_{k \in k 2} \sum_{i \in N} c_{i 0} x_{i 0}^{k}
$$

With constraints:

1. Every customer is visited exactly once and only by one vehicle.

$$
\sum_{k \in K} \sum_{\substack{i \in V \\ i \neq j}} x_{i j}^{k}=1, \quad \forall j \in N
$$

2. Every vehicle that visits a customer, after finished serving will immediately leave the customer.

$$
\sum_{\substack{i \in V \\ i \neq j}} x_{i j}^{k}-\sum_{\substack{i \in V \\ i \neq j}} x_{j i}^{k}=0, \quad \forall j \in V, \forall k \in K
$$

3. The total requests of each customer on the route taken by each transport vehicle not exceed the capacity.

$$
\sum_{j \in N} q_{j}\left(\sum_{i \in V} x_{i j}^{k}\right) \leq \text { Maxcapacity, } \quad \forall k \in K
$$

4. Only one vehicle will depart from the depot to serve one route.

$$
\sum_{i \in N} x_{0 i}^{k} \leq 1, \quad \forall k \in K
$$

5. Private vehicles owned by companies are limited to $\mathrm{Nu}$. 


$$
\sum_{k \in K 1} \sum_{i \in N} x_{0 i}^{k}=N u
$$

6. Defining the variable $x$ for each vehicle $k$.

$$
x_{i j}^{k} \in\{0,1\} \quad \forall i, j \in V, \forall k \in K, i \neq j
$$

Meanwhile, the decision variable are:

$$
x_{i j}^{k}= \begin{cases}1, & \text { if vehicle } k \text { serves } j \text { after serving } i \\ 0, & \text { Otherwise }\end{cases}
$$

where

$$
\begin{array}{ll}
c_{i j} & : \text { Travel costs from customer } i \text { to customer } \\
c_{i 0} & : \text { Travel costs from customer } i \text { to depot } \\
F_{k} & : \text { Fixed cost for vehicles } k \\
K & : \text { The set of vehicles, with } \mathrm{K}=\{K 1 \cup K 2\} \\
K 1 & : \text { The set of private vehicles } \\
K 2 & : \text { The set of rental vehicles } \\
\text { MaxCap } & : \text { Maximum capacity of the vehicle } \\
N u & : \text { Maximum number of private vehicles } \\
q_{j} & : \text { Amount of requests from customers } j \\
V & : \text { The set of points } \\
x_{i j}^{k} & : \text { Vehicle } k \text { that serves customer } j \text { after visiting customer } i \\
x_{i 0}^{k} & : \text { Vehicle } k \text { that returns to the depot after visiting customers } i \\
x_{0 i}^{k} & : \text { Vehicle } k \text { that serves customer } i \text { right after vehicle departs from the }
\end{array}
$$

\section{Bat Algorithm}

Bat algorithm is an algorithm that is adopted from the microbat's behavior when it preys, determines the perch location and avoids obstacles when flying by using sound waves it releases, which is called echolocation.

There are three approaches used in bat algorithm:

a. All bats use echolocation to estimate distances and can distinguish between prey and obstacles.

b. Flying bats (moving) with speed $v_{i}$ dan posistion $x_{i}$ that varying with a fixed minimum frequency $\left(Q_{\min }\right)$ also loudness $A_{o}$ varied in finding prey. The bat can automatically adjust the frequency of the sound waves it emits and adjust pulse rate $\left(r^{0} \in[0,1]\right)$ depends on the proximity of the target.

c. Although the loudness of various bats is assumed that starting from $A_{o}$ in the form of a positive value that is large enough to the minimum $A_{\text {min }}$. 


\subsection{Echolocation}

Bats are the only mammals that have wings and also have the ability called echolocation. It is estimated that there are 1000 species of bat species. Bats with arms measuring 2.2$11 \mathrm{~cm}$ are called small bats (microbat). Most bats use echolocation and microbat is a type of bat that is often used sample due to the most intensive use echolocation than other types.

Most microbat is insectivorous (insective) and uses sound waves emitted called echolocation to detect prey, avoid obstacles while flying and determine the location of perches when there is no light. The reflection of the sound waves that it emits will be recaptured by bats, thus bats can detect objects around them.

The sound waves that it emits varying depends on the target and its species and bats tend to use a constant frequency. Some types of bats have good vision and sharp sense of smell, but they still use echolocation when flying or combine the sense of sight and smell.

\subsection{Movement of Bats}

Each bat depends on speed $v_{i}^{t}$ and position $x_{i}^{t}$ by adjusting i on iteration $t$ on the solution. Among all the bats there is one solution while the best global position $x_{\text {best }}$. update the position and speed of each bat in the iteration $t$ three formulas are used as follows:

$$
\begin{aligned}
& Q_{\mathrm{i}}=Q_{\min }+\left(Q_{\max }-Q_{\min }\right) * \beta_{i} \\
& v_{i, j}^{t+1}=v_{i, j}^{t}+\left(x_{i, j}^{t}-x_{\text {best }}\right) * Q_{i} \\
& x_{i, j}^{t+1}=x_{i, j}^{t}+v_{i, j}^{t+1}
\end{aligned}
$$

where: bat_size)

$J \quad=$ index of the number of candidate solutions $(i=1,2, \ldots$, bat_size)

$Q_{i} \quad=$ bat $i$ frequency

$Q_{\min } \quad=$ minimum bat frequency

$Q_{\max } \quad=$ maximum bat frequency

$\beta_{i} \quad=$ Real numbers generated randomly at bat $i$ at interval $[0,1]$

$x_{\text {best }} \quad=$ the best temporary position of the bat

$x_{i, j}^{t} \quad=$ bat position during iteration $t$

$x_{i, j}^{t+1} \quad=$ bat position during the next iteration 


$$
\begin{array}{ll}
v_{i, j}^{t} & =\text { bat speed during iteration } \mathrm{t} \\
v_{i, j}^{t+1} & =\text { bat speed during the next iteration }
\end{array}
$$

For simplicity, the value $\beta$ which is a real number that is randomly generated at intervals $[0,1]$ is assumed to be $Q \epsilon\left[Q_{\min }, Q_{\max }\right]$ with $Q_{\min }=0$ and $Q_{\max }=1$ [6]

\subsection{Local Search}

In the process of local search, it starts with determining the best solution of each bat that matches or is often referred to as personal-best-bat $t_{i}\left(x_{\mathrm{p}}\right)$. he new solution is sought based on a formula called random walk, as follows:

$$
x_{\text {new }}=x_{p}+\varepsilon A^{t}
$$

where:

$$
\begin{array}{ll}
x_{\text {new }} & =\text { bat position from local search results } \\
x_{p} & =\text { best local position for each bat } \\
\varepsilon & =\text { real numbers from random numbers between }-1 \text { and } 1 \\
A^{t} & =\text { the average loudness of each bat in the iteration } t
\end{array}
$$

\subsection{Loudness and Pulse Rate Emission}

Each bat has a different loudness $\left(A_{i}\right)$ and pulse rate $\left(r_{i}\right)$ and will be updated according to the iteration process. Changes in and $r_{i}$ according to the following formula:

$$
\begin{aligned}
A_{i}^{t+1} & =\propto A_{i}^{t} \\
r_{i}^{t+1} & =r_{i}^{0}[1-\exp (-\gamma t)]
\end{aligned}
$$

where:

$$
\begin{array}{ll}
\propto & =\text { the coefficient of decreasing loudness } 0<\propto<1 \\
\gamma & =\text { the coefficient of the increase in pulse rate in } \gamma>0 \\
A_{i}^{t+1} & =\text { loudness- } i \text { at the next } t \text { iteration } \\
A_{i}^{t} & =\text { loudness- } i \text { at the time of iteration } t \\
r_{i}^{t+1} & =\text { pulse rate- } i \text { at the next iteration } t \\
r_{i}^{0} & =\text { pulse rate- } i \text { during initial iteration }
\end{array}
$$

\section{COMVRP Using Bat Algorithm Procedure}

There are several steps to solve COMVRP using Bat Algorithm: 
1. Input the number of customers, depot location data, customer location data, maximum vehicle capacity, maximum number of private vehicles, private vehicle fixed costs, rental vehicle costs, travel costs and the number of requests per customer.

2. Determine the parameters BA, namely the number of bat, maximum iteration, $\alpha=$ $0.9, \gamma=0.9$, minimum frequency $=0\left(Q_{\min }\right)$, maximum frequency $=1\left(Q_{\max }\right)$, initial pulse rate $\left(r_{o}\right)$ and initial loudness $=1\left(A_{o}\right)$.

3. Form the initial bat population with:

i. Generate random real numbers at intervals $[0,1]$ as many as the number of customers (n) that will represent the start position of each bat $\left(x_{i}\right)$ and generate as many bats (batsize).

ii. Generate random real numbers at intervals $[0,1]$ as many as the number of customers $(\mathrm{n})$ which will represent the initial velocity of each bat $\left(v_{i}\right)$ and generate as many bats (batsize).

4. Evaluate the objective function

i. Sorting the results of the generation of real random numbers that represent $x_{i}$.

ii. Displays the serial number of the results of the generation of random real numbers that have been sorted on each $x_{i}$ so that a candidate route is formed

iii. Determine the route traveled by vehicles with obstacles including each trip that uses a private vehicle route starts and ends at the depot, every trip using a vehicle rental route starts at the depot and ends at the last customer and then the total load of each vehicle may not exceed the maximum capacity of the vehicle.

iv. Calculates the value of the objective function $f\left(x_{i}\right)$ from COMVRP.

v. Compares the objective function value of each bat $f\left(x_{i}\right)$ to determine the best temporary solution $f\left(x_{\text {best }}\right)$.

5. Update the position of each bat $\left(x_{i}\right)$ based on the new frequency and speed of each bat $\left(v_{i}\right)$. The steps are as follows :

i. $\quad$ Determine the bat frequency value $\left(Q_{\mathrm{i}}\right)$.

ii. Determine the new velocity $\left(v_{i}\right)$.

iii. Determine the new position of each bat $\left(x_{i}\right)$.

iv. Update the position of each bat from the update.

6. Evaluate the objective function of the bat to renew the position.

7. For each bat the process is as follows :

i. Compare the pulse rate of each bat $\left(r_{i}\right)$ with real numbers generated randomly at intervals $[0,1]$. If rand number is $a c a k_{i}>r_{i}$ then find the best solution bat $_{i}\left(x_{p}\right)$ but if not then go directly to step $\mathrm{v}$ to evaluate the objective function.

ii. Do a local search around the best local bat $x_{p}$.

iii. Replaces the new position with the position of the local search results. 
iv. Evaluating the objective function from local search results.

v. Compare the new objective function with the old objective function. If new $f\left(x_{i}^{t}\right)<$ old $f\left(x_{i}^{t}\right)$, then accept the new $f\left(x_{i}^{t}\right)$ and move on to the next step. But if not, then reject the new $f\left(x_{i}^{t}\right)$ and make the previous position or the old $f\left(x_{i}^{t}\right)$ as the new position.

vi. Compare loudness bat $\left(A_{i}\right)$ with real numbers generated randomly at intervals [0,1]. If $A_{i}$ is more than the value of a real number that is randomly generated at intervals $[0,1]$, then it updates $r_{i}$ and $A_{i}$. However, if $A_{i}$ is no more than the random number, then make the previous position or the old $f\left(x_{i}^{t}\right)$ as the new position.

vii. If all bat $(x i)$ has been processed then go to the next step, but if not return to the step 7.

8. Save the new solutions, $A_{i}$ and $r_{i}$ each bat and find the best solution among new bat solutions.

9. Check iteration, If the iteration equals the maximum iteration then the iteration stops and makes the best global solution in the last iteration as the final solution in the process of solving a COMVRP problem. If it has not been achieved, then return to step 5 .

\section{$5 \quad$ Result and Discussion}

In this problem there are two types of data used, the first is distance data obtained from http://www.coin.or.org/SYMPHONY/branchandcut/VRP/data/ index.htm.old/. Distance data taken consists of 3 types, namely data from small data (18 customers), medium data ( 75 customers), and big data (100 customers). Then the second is cost data [7]. There are two kinds of data taken from these sources, namely the cost per unit of distance and the rental fee of each vehicle.

The parameters used in the completion of COMVRP with 18 customer data are initial pulse rate $\left(r_{o}\right)=0.3 ; 0.6 ; 0.9$, bat size $=10 ; 50 ; 100$, maximum iteration (MaxIter) $=$ $10 ; 100 ; 1000$, Maximum number of private vehicles $(\mathrm{Nu})=2$, MaxCapacity $=85$, Fixed cost of private vehicle $\left(F_{0}\right)=1 €$, Fixed cost rental vehicle $\left(F_{1}\right)=15 €$, Cost for each distance $=0,6 €$, minimum frequency $\left(Q_{\min }\right)=0$, maximum frequency $\left(Q_{\max }\right)=1, \alpha=$ $0.9, \gamma=0.9$ and initial loudness $\left(A_{o}\right)=1$. The final results obtained are presented in Table 1.

The best results obtained from small data with 18 customers are $183.79 €$ with a maximum iteration of 1000 , the number of bat 50 and the initial pulse rate $\left(r_{o}\right)$ is 0.3 . 
Table 1 Results of Programs on Small Data

\begin{tabular}{|c|c|c|c|c|}
\hline \multirow{2}{*}{ MaxIter } & \multirow{2}{*}{ Bat size } & \multicolumn{3}{|c|}{$\boldsymbol{r}_{\boldsymbol{o}}$} \\
\cline { 3 - 5 } & & $\mathbf{0 . 3}$ & $\mathbf{0 . 6}$ & $\mathbf{0 . 9}$ \\
\hline \multirow{3}{*}{10} & 10 & 243,07 & 250,43 & 248,27 \\
\cline { 2 - 5 } & 50 & 246,93 & 246,37 & 263,55 \\
\cline { 2 - 5 } & 100 & 254,11 & 242,11 & 248,39 \\
\hline \multirow{3}{*}{100} & 10 & 246,19 & 244,93 & 236,96 \\
\cline { 2 - 5 } & 50 & 229,20 & 242,30 & 236,67 \\
\cline { 2 - 5 } & 100 & 225,30 & 238,52 & 245,45 \\
\hline \multirow{3}{*}{1000} & 10 & 229,92 & 208,23 & 222,92 \\
\cline { 2 - 5 } & 50 & $\underline{\mathbf{1 8 3 , 7 9}}$ & 204,40 & 240 \\
\cline { 2 - 5 } & 100 & 204,67 & 211,17 & 210,53 \\
\hline
\end{tabular}

Parameters used in the completion of COMVRP with 75 customer data are initial pulse rate $\left(r_{o}\right)=0.3 ; 0.6 ; 0.9$, bat size $=10 ; 50 ; 100$, maximum iteration $($ MaxIter $)=10 ; 100 ; 1000$, Maximum number of private vehicles $(\mathrm{Nu})=2$, MaxCapacity $=250$, Fixed cost of private vehicle $\left(F_{0}\right)=1 €$, Fixed cost rental vehicle $\left(F_{1}\right)=15 €$, Cost for each distance $=0,6 €$, minimum frequency $\left(Q_{\min }\right)=0$, maximum frequency $\left(Q_{\max }\right)=1, \alpha=0.9, \gamma=0.9$ and initial loudness $\left(A_{o}\right)=1$. The final results obtained are presented in Table 2 .

Table 2 Results of Programs on Medium Data

\begin{tabular}{|c|c|c|c|c|}
\hline \multirow{2}{*}{ MaxIter } & \multirow{2}{*}{ Bat size } & \multicolumn{3}{|c|}{$\boldsymbol{r}_{\boldsymbol{o}}$} \\
\cline { 2 - 5 } & & $\mathbf{0 . 3}$ & $\mathbf{0 . 6}$ & $\mathbf{0 . 9}$ \\
\hline \multirow{3}{*}{10} & 10 & 1369,21 & 1380,79 & 1395,76 \\
\cline { 2 - 5 } & 50 & 1305,12 & 1356,46 & 1370,16 \\
\cline { 2 - 5 } & 100 & 1232,83 & 1306,59 & 1312,25 \\
\hline \multirow{3}{*}{100} & 10 & 1339,01 & 1352,31 & 1318,02 \\
\cline { 2 - 5 } & 50 & 1248,97 & 1271,22 & 1259,05 \\
\cline { 2 - 5 } & 100 & 1226,36 & 1308,73 & 1244,21 \\
\hline \multirow{3}{*}{1000} & 10 & 1176,03 & $\mathbf{1 1 4 5 , 0 5}$ & 1228,77 \\
\cline { 2 - 5 } & 50 & 1166,04 & 1221,39 & 1204,37 \\
\cline { 2 - 5 } & 100 & 1206,93 & 1189,23 & 1162,09 \\
\hline
\end{tabular}

The best results obtained from medium data with 75 customers are $1145.05 €$ with a maximum iteration of 1000 , the number of bat 10 and the initial pulse rate $\left(r_{o}\right)$ is 0.6.

The parameters used in the completion of COMVRP with 100 customer data are initial pulse rate $\left(r_{o}\right)=0.3 ; 0.6 ; 0.9$, bat size $=10 ; 50 ; 100$, maximum iteration (MaxIter) $=$ 10;100;1000, Maximum number of private vehicles $(\mathrm{Nu})=2$, MaxCapacity $=300$, Fixed cost of private vehicle $\left(F_{0}\right)=1 €$, Fixed cost rental vehicle $\left(F_{1}\right)=15 €$, Cost for each 
distance $=0,6 €$, minimum frequency $\left(Q_{\min }\right)=0$, maximum frequency $\left(Q_{\max }\right)=1, \alpha=$ $0.9, \gamma=0.9$ and initial loudness $\left(A_{o}\right)=1$. The final results obtained are presented in Table 3.

The best results obtained from big data with 100 customers are $€ 1511.80$ with a maximum iteration of 1000 , the number of bat 100 and the initial pulse rate $\left(r_{o}\right) \mathrm{b}$ is 0.3 . The best solution of large sample data cases based on the best results obtained can be seen in Table 4.

Table 3. Results of Programs on Large Data

\begin{tabular}{|c|c|c|c|c|}
\hline \multirow{2}{*}{ MaxIter } & \multirow{2}{*}{ Batsize } & \multicolumn{3}{|c|}{$\boldsymbol{r}_{\boldsymbol{o}}$} \\
\cline { 3 - 5 } & & $\mathbf{0 . 3}$ & $\mathbf{0 . 6}$ & $\mathbf{0 . 9}$ \\
\hline \multirow{3}{*}{10} & 10 & 1917,59 & 1867,76 & 1897,00 \\
\cline { 2 - 5 } & 50 & 1748,00 & 1835,96 & 1843,15 \\
\cline { 2 - 5 } & 100 & 1847,49 & 1820,49 & 1862,30 \\
\hline \multirow{3}{*}{100} & 10 & 1819,22 & 1825,88 & 1710,48 \\
\cline { 2 - 5 } & 50 & 1774,39 & 1691,25 & 1748,27 \\
\cline { 2 - 5 } & 100 & 1770,40 & 1820,76 & 1778,26 \\
\hline \multirow{3}{*}{1000} & 10 & 1629,45 & 1639,37 & 1739,65 \\
\cline { 2 - 5 } & 50 & 1661,22 & 1683,57 & 1559,05 \\
\cline { 2 - 5 } & 100 & $\underline{\mathbf{1 5 1 1 , 8 0}}$ & 1614,51 & 1674,93 \\
\hline
\end{tabular}

Table 4 Best Solution on Large Data

\begin{tabular}{|c|l|c|}
\hline Vehicle & \multicolumn{1}{|c|}{ Route } & \multicolumn{1}{|c|}{ Total Cost } \\
\cline { 1 - 2 } 1 & $\begin{array}{l}0-14-42-16-36-46-17-23-26-13-50-10-88-63-51-91-61- \\
86-5-40-98-31-0\end{array}$ & \multirow{2}{*}{1511,80} \\
\cline { 1 - 2 } 2 & $\begin{array}{l}0-80-4-8-93-53-78-27-67-43-97-44-96-6-74-72-29-54- \\
56-20-32-90-41-75-25-24-92-0\end{array}$ & \\
\cline { 1 - 2 } 3 & $\begin{array}{l}0-70-3-35-89-100-28-48-18-47-59-95-12-81-34-33-11- \\
15-57-55\end{array}$ & \\
\hline 4 & $0-37-38-85-39-22-52-83-94-87-58-62-7-84-45-19-69-2$ \\
\cline { 1 - 2 }
\end{tabular}

\section{Conclusion}

Implementation of the program for example cases using small data obtained the best solution with a total cost of $183.79 €$, with medium size data obtained the best solution with a total cost of $1145.05 €$, with large data obtained the best solution with a total cost of $1511.80 €$. The pattern of the results of the running program shows that the more 
iterations, the smaller the total costs are obtained, while for pulse rate $\left(r_{o}\right)$ and the amount of bat the value are tends not to affect the total cost obtained.

\section{$7 \quad$ References}

[1] Batubara, S., Maulidya, R., and Kusumaningrum, I., 2013, Perbaikan Sistem Distribusi dan Transportasi dengan Menggunakan Distribution Requirement Planning (DRP) dan Alogaritma Djikstra, Jurnal Teknik Industri, 1411-6430.

[2] Belfiore, P., Tsugunobu, H., and Yoshizaki, Y., 2008, Scatter Search for Vehicle Routing Problem with Time Windows and Split Deliveries, Vehicle Routing Problem, 1, 1-14

[3] Bräysy, O., 2001. Genetic Algorithms for the Vehicle Routing Problem with Time Windows, Arpakannus Special Issue on Bioinformatics and Genetic Algorithms, $\mathbf{1}$, 33-38.

[4] Liu, R. and Jiang, Z., 2012, The Close-Open Mixed Vehicle Routing Problem. European Journal of Operational Research, 220, 349-360.

[5] Yang, X.S., 2010, A New Metaheuristic Bat-Inspired Algorithm, Nature Inspired Cooperatives Strategies for Optimization, Springer, Berlin, 284, 65-74.

[6] Yang, X.S., 2013, Bat Algorithm: Literature Review And Applications, Int. J. BioInspired Computation, 5, 141-149.

[7] Čičková, Z., Brezina, I., dan Pekár, J., 2015, Cost Analysis of Open VRP, Logic $2^{\text {nd }}$ Logistic International Conference, 8-13 